\title{
A Quantitative Assessment of Microtexture in Titanium Alloys using Destructive and Nondestructive Methods
}

\author{
Adam L. Pilchak ${ }^{1}$, Jia Li $^{2}$, Gaofeng Sha ${ }^{2}$, Michael Groeber ${ }^{1}$, Joseph Tucker ${ }^{3}$ and Stan Rokhlin ${ }^{2}$ \\ 1. Air Force Research Laboratory, Materials and Manufacturing Directorate, WPAFB, OH USA. \\ 2. The Ohio State University, Department of Materials Science and Engineering, Columbus, OH USA \\ 3. UES, Inc., Dayton, OH USA
}

Due to the need to balance high performance with safety, ultrasonic inspection is routinely performed on many critical aerospace components at various stages of manufacturing. For instance, cylindrical titanium billets are inspected to look for nitrogen-stabilized defects (hard alpha). After forging, the material is machined into its sonic shape which enables rapid analysis using scanning ultrasound (or phased array) systems. While these inspections are generally pass/fail with very little additional information being stored or utilized, there is actually a wealth of information in the ultrasound data related to microstructure. In the case of titanium alloys, mostly owing to the two-phase structure and elastic anisotropy, the microstructure evolution mechanisms are generally of the non-classical type, i.e. there is no classical nucleation and growth of high angle grain boundaries, but rather dislocation climb and glide result in continuous evolution of the microstructure and texture from the initial to final condition. Based on this knowledge, Semiatin and co-workers [1] have developed a series of microstructure and texture evolution models for sub-ß-transus worked two-phase titanium alloys. The final critical aspect to consider in titanium alloys is the effect of microtexture, or large clustered regions of primary alpha particles of similar orientation. These microtextured regions (MTRs) are particularly deleterious to dwell fatigue properties in near-alpha titanium alloys, but also impact fatigue lifetime variability in general as well. From the perspectives of location specific design and serial-number based lifing, it would be beneficial to develop quantitative relationships between the size, shape, spatial orientation, crystallographic orientation and range of alpha particle c-axis misorientations within each MTR which all have first order effects on dwell fatigue properties [2]. In this paper, we describe a method to obtain size, shape and an effective one-dimensional orientation distribution for MTRs (spread in alpha particle size) by inversion of ultrasound attenuation and backscattering data collected in three-directions. The results are compared to similar measurements obtained from EBSD data.

The material used in the present study was $152-\mathrm{mm}$ diameter Ti-6Al-2Sn-4Zr-2Mo-0.1Si billet in the alpha+beta worked condition. The microstructure and microtexture of the material has been previously described [3]. Briefly, the material contained MTRs with their primary axis elongated parallel to the billet axis. There was a gradient in MTR sizes with those near the outer diameter being fine and of few in number while there were many coarse MTRs in the center. A sample measuring $66 \times 67 \times 115 \mathrm{~mm}$ was extracted off-center from the billet axis such that the two edges measuring $67 \mathrm{~mm}$ were near the outer diameter and center of the billet such that there was a gradient in MTRs in the $66 \mathrm{~mm}$ direction. Samples for electron backscatter diffraction were extracted at OD, mid-radius and edge locations in longitudinal and transverse orientations. Ultrasonic attenuation and backscattering data were collected in the three principal sample directions and processed as described in [4]. The ultrasound data were inverted by the methods described in [5] to obtain spatially resolved, volume averaged ellipsoidal representations of the MTRs. This inversion uses a generalized version of Poisson's geometrical autocorrelation function which describes the probability that the head and tail of a vector placed arbitrarily in the microstructure will have the same crystallographic orientation. Here, because ultrasonic 
and mechanical properties depend most heavily on c-axis orientation and are less sensitive to rotations about this direction, we have calculated the autocorrelation function of c-axes from the EBSD data.

The variation in microtextured region size, shape and intensity in the sample is illustrated in Figure 1. From these crystal orientation maps, it is evident that all locations exhibit high aspect ratio MTRs, though their size and shape vary throughout the billet. The autocorrelation of c-axes for each location, in the axial and radial directions, are also shown in Figure 1. The measured autocorrelations, $\mathbf{S}_{2}^{c}(\mathbf{r})$, were fit to a form of the geometric autocorrelation function used in the inverse scattering model which included an additional term to account for short-range correlations present in EBSD. Under the assumption of Poisson statistics, the decay constant in the exponentially decaying distribution is equivalent to the [6], the average MTR cord length (average MTR intercept length) and two-times the decay constant is equal to the average MTR size. The results of the ultrasound and EBSD measurements are shown in Table 1. The volume averaged MTR sizes obtained via ultrasound are slightly larger and smaller than those measured by EBSD in the axial and radial directions, respectively. The assumptions of the model and the sources of variability in the measurements will be discussed in more detail during the presentation.

In conclusion, the feasibility of measuring MTR sizes in titanium alloys both destructively and nondestructively has been demonstrated. Additional effort is needed to further refine the MTR quantification routines and additional characterization is required to make a more direct comparison of the two methods.
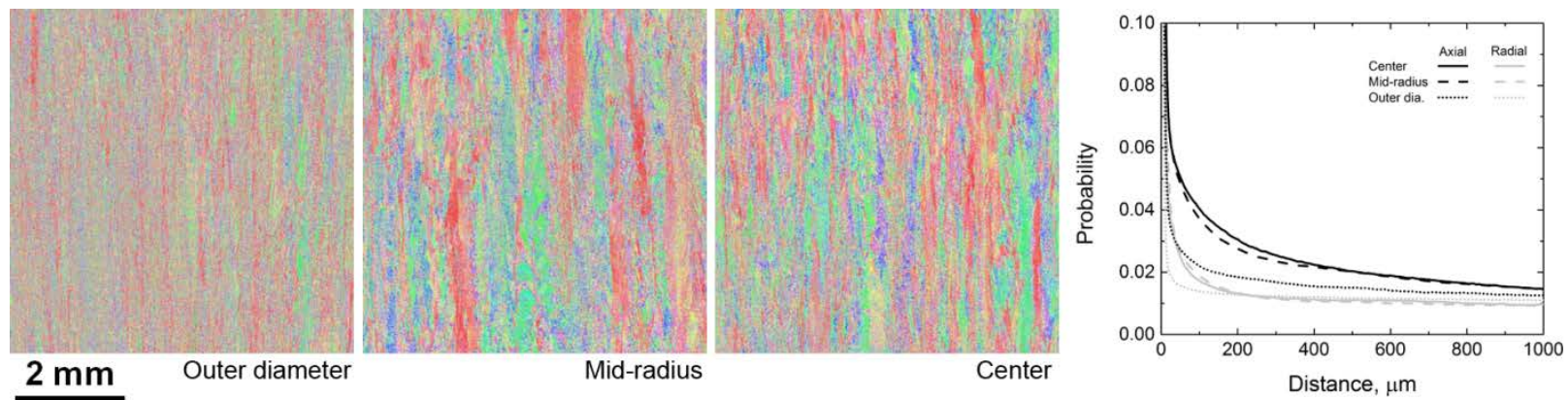

Figure 1. Crystal orientation maps for various longitudinal sections of the billet and their autocorrelation functions in the axial and radial directions.

Table 1. Comparison of EBSD and ultrasound measured MTR sizes.

\begin{tabular}{|l|c|c|r|c|}
\hline & \multicolumn{2}{|c|}{ EBSD } & \multicolumn{2}{c|}{ Ultrasound } \\
\hline & Axial & Radial & Axial & Radial \\
\hline Outer diameter & 408 & 112 & 614 & 100 \\
\cline { 1 - 3 } Mid-radius & 564 & 222 & & \\
\cline { 1 - 3 } Center & 481 & 246 & & \\
\hline
\end{tabular}

References:

[1] D. Furrer and S.L. Semiatin, ASM Handbook vol. 22A \& 22B, ASM International, 2010.

[2] A.P. Woodfield et al, Titanium '95: Science and Technology (1996) p. 1116.

[3] A.L. Pilchak et al, Metall. Trans. A 44 (2013), p. 4881.

[4] O.I. Lobkis et al, Ultrasonics 52 (2012) p. 594.

[5] J. Li et al, in QNDE 31 (2012) p. 1397.

[6] F. Stanke, J. Acoust. Soc. Am. 80 (1986) p. 1479. 